\title{
NILPOTENT INJECTORS IN FINITE GROUPS
}

\author{
Peter FBrster
}

Nilpotent injectors exist in all finite groups.

For every Fitting class $F$ of finite groups (see [2]), Inj $F(G)$ denotes the set of all $H \leq G$ such that for each $N \forall \forall G, H \cap N$ is an F-maximal subgroup of $N$ (that is, belongs to $F$ and is maximal among the subgroups of $N$ with this property). Let $N$ and $N^{*}$ denote the Fitting class of all nilpotent and quasi-nilpotent groups, respectively. (For the basic properties of quasi-nilpotent groups, and of the $N^{*}$-radical $F^{*}(G)$ of a finite group $G$, the reader is referred to [5],X. 13 ; we shall use these properties without further reference.) Blessenohl and H. Laue have shown in [1] that for every finite group $G$, $\operatorname{Inj}_{N^{*}}(G)=\left\{H \leq G \mid H \geq F^{*}(G) N^{*}\right.$-maximal in $\left.G\right\}$ is a non-empty conjugacy class of subgroups of $G$. More recently, Iranzo and Pérez-Monasor have verified $\operatorname{Inj}_{N}(G) \neq \varnothing$ for all finite groups $G$ satisfying $G=C_{G}(E(G)) E(G)$ (see $\left.[6]\right)$, and have extended this result to a somewhat larger class $M$ of finite groups $C$ (see [7]). One checks, however, that $M$ does not contain all finite groups; for example, $S_{5} \notin M$. Here we shall apply a result of Glauberman [4] together with the Feit-Thompson Theorem to derive from the Blessenohl-Laue result the following

Received 27 February 1985

Copyright Clearance Centre, Inc. Serial-fee code: 0004-9727/85 $\$ A 2.00+0.00$. 
THEOREM. For every finite group $G$ and every $T / Z(E(G)) \in S y z_{2}(E(G) / Z(E(G))$ ),

$$
\not \neq \operatorname{Inj}_{N^{*}}\left(N_{G}(T)\right) \subseteq \operatorname{Inj}_{N^{(G)}} .
$$

Proof. To begin with, we shall prove:

(*) $H \in \operatorname{Inj}_{N^{*}}\left(N_{G}(T)\right) \Rightarrow H \in N$; in particular, $H \in \operatorname{Inj}_{N}\left(N_{G}(T)\right)$.

Since $H=F^{*}(H)=E(H) F(H)$ (a central product), it suffices to show that $E(H)=1$. The nilpotent (and thus quasi-nilpotent) nomal subgroup $T$ of $N_{G}(T)$ is contained in $F(H)$. Similarly, $F(G) \leq C_{G}(E(G)) \leq C_{G}(T) \leq N_{G}(T)$ gives $E(G) \leq F(H)$. Hence

$$
E(H) \leq C_{H}(F(H)) \leq C_{H}(T F(G)) .
$$

Put $Z=Z(E(G)), C=C_{G}(E(G) / Z), \quad D=C_{G}(T / Z) \geq C$. We have just shown that $D \geq E(H)$. As $E(G) / Z$ is a di rect product of non-abelian simple groups, we have that (to within isomorphism) $D / C$ is a subgroup of $C_{A u t(E(G) / Z)}(T / Z)$, which (in view of $T / Z \in S y Z_{2}(E(G) / Z)$ ) by a result of Glauberman [4], is a 2-nilpotent-group; note that $O_{2},(E(G) / Z)=1$ follows from the Feit-Thompson Theorem. Hence $E(H) C / C$ is 2-nilpotent and, by the Feit-Thompson Theorem again, is soluble. We conclude that the perfect group $E(H)$ is contained in $C$. As $Z(E(G))=\Phi(E(G))$, our Proposition below therefore shows that $E(H) / C_{E(H)}(E(G))$ is nilpotent. Now perfectness of $E(H)$ yields that $E(H) \leq C_{G}(E(G))$. Together with the above observation this gives

$$
E(H) \leq C_{G}(E(G)) \cap C_{G}(F(G))=C_{G}\left(F^{*}(G)\right)=2\left(F^{*}(G)\right) .
$$

From this together with perfectness of $E(G)$ again, the desired conclusion that $E(H)=1$ is immediate.

Using induction on $|G|$, we can now prove that $H \in \operatorname{Inj}_{N}(G)$.

$$
H \leq X \leq G, X \in N \Rightarrow H=X:
$$

Clearly, $T / Z \in S y Z_{2}(X / Z \cap E(G) / Z)$, whence $X / Z \cap E(G) / Z \exists X / Z \in N$ implies that $T / Z=O_{2}(X / Z \cap E(G) / Z) \forall X / Z$; that is, $X \leq N_{G}(T)$.

Thus (1) follows from (*).

(2) If $N$ is a maximal normal subgroup of $G$, then $B \cap N \in \operatorname{Inj}{ }_{N}(N)$ : 
By the inductive hypothesis, it suffices to show that $H \cap N \in \operatorname{Inj}_{N^{*}}\left(N_{N}(S)\right)$ for some $S \geq 2(E(N))$ such that $S / Z(E(N)) \in S y z_{2}(E(N) / Z(E(N)))$. Now note that $\quad H \cap N \in \operatorname{Inj} N_{N^{*}}\left(N_{N}(T)\right)$. Therefore, if $E(G) \leq N$ (in which case $E(N)=E(G)$ ), we may choose $S=T$, proving our claim. Suppose, then, that $E(G) \neq N$. Then $G=N E(G)$, and $E(G)$ is a central product of $E(N) Z=E(G) \cap N$ and $E Z$ for some component $E$ of $E(G)$. Let $S=T \cap E(N)$. Then from $Z \cap E(N)=Z(E(N))$ we infer that $S / Z(E(N)) \in S y Z_{2}(E(N) / Z(E(N)))$. Moreover,

$$
N_{N}(T) \leq N_{N}(T \cap E(N))=N_{N}(S) \leq N_{N}(S R)=N_{N}(T),
$$

where $R=T \cap E Z$; observe that $T / Z=(T \cap E(N) Z) / Z \times(T \cap E Z) / Z$ and $[N, R] \leq[N, E Z] \leq N \cap E Z=Z \leq R$. Hence $N_{N}(T)=N_{N}(S)$, and the proof of (2) is complete.

Finally, $H \in \operatorname{Inj}_{N}(G)$ follows from $(I+2)$.

The second half of the above proof yields the following COROLLARY. Let $T / Z(E(G)) \in S y z_{p}(E(G) / Z(E(G)))$. Then

$$
\left.\operatorname{Inj}_{N}\left(N_{G}(T)\right) \subseteq \operatorname{Inj}{ }_{N}(G)\right) .
$$

Note that for $p \nmid|E(G) / Z(E(G))|, N_{G}(T)=G$. Furthermore, it is not in general true that $\operatorname{Inj}_{N}\left(N_{G}(T)\right)$ is a single conjugacy class of subgroups of $G$, as is shown by taking $G=A_{12}(=E(G))$ and $I \in S y Z_{7}(G)$; also, in this example, $\operatorname{Inj} j_{N}\left(N_{G}(T)\right) \cap I n j_{N^{*}}\left(N_{G}(T)\right)=\varnothing$, for $A_{5} \times T \cong C_{G}(T) \in \operatorname{Inj}_{N^{*}}\left(N_{G}(T)\right)$.

For any finite group $G$, let $F^{\prime}(G)=S(G \bmod \Phi(G))$, where $S(X)$ denotes the socle of $X$. Clearly, $\left.F^{*}(G) \leq F^{\prime}(G)\right)$ and $F^{\prime}\left(F^{*}(G)\right)=F^{*}(G)$. In our proof of the above Theorem we have applied the following 'global' version of $[3], 1.2 b$ :

PROPOSITION. Suppose that for some finite group $G$ and some $A \leq \operatorname{Aut}(G)$, we have $\left[A, F_{i}\right] \leq F_{i-1}(i=1, \ldots, n)$, where the $F_{i} \forall G$ are such that

$$
\Phi(G)=F_{0} \leq E_{1} \leq \ldots \leq F_{n}=F^{\prime}(G) .
$$

Then $A$ is a nilpotent $\pi\left(F^{\prime}(G)\right)$-group. 
Proof. Consider the semidirect product $H=A \bar{G}$, where $\bar{G}=G / \Phi(G)$. By $[3], 1.1, C_{\bar{G}}\left(F^{\prime}(\bar{G})\right) \leq F^{\prime}(\bar{G})$, and so we obtain from Dedekind's modular law

$$
\begin{aligned}
A F^{\prime}(\bar{G})= & A C_{\bar{G}}\left(F^{\prime}(\bar{G})\right) F^{\prime}(\bar{G})=C_{H}\left(F^{\prime}(\bar{G})\right) F^{\prime}(\bar{G}) \triangleleft H, \\
& {[A, G] \leq A F^{\prime}(G) \cap G=F^{\prime}(G) . }
\end{aligned}
$$

This together with $\left[A, F_{i}\right] \leq E_{i-1}$ shows that $A$ centralises each chief factor of $G / \Phi(G)$. Since the latter condition is inherited by $A / C_{A}\left(G / O_{p}(G)\right),\left(G / O_{p}(G)\right.$ from $A, G$, we may apply $[3], 1.2 b$ to conclude that for every prime $p$

$$
A / C_{A}\left(G / O_{p},(G)\right) \text { is a } p \text {-group. }
$$

If $\pi$ denotes $\pi\left(F^{\prime}(G)\right)$, then from $S(G) \leq F^{\prime}(G)$ we get

$$
n_{p \in \pi} O_{p},(G)=1 \text {. }
$$

Consequently, $A \cong A / \cap{ }_{p \in \pi} C_{A}\left(G / O_{p},(G)\right)$ is a nilpotent $\pi$-group.

Notice that a somewhat more careful argument in the above proof would have shown that $[A, G] \leq F(G)$, yielding that $A$ is a $\pi(F(G))$ group.

\section{References.}

[1] D. Blessenohl, H. Laue, "Fittingklassen endlicher Gruppen, in denen gewisse Hauptfaktoren einfach sind", J. AZgebra 56, (1979) , 516-5 32 .

[2] B. Fischer, w. Gaschütz, B. Hartley, "Injektoren endlicher auflösbarer Gruppen". Math. 2. 102, (1967), 337-339.

[3] P. Förster, "Projektive Klassen endlicher Gruppen. IIa." Pubz.Sec.Mat.Univ.Aut.BarceZona 29, (1985). (In the press.)

[4] G. Glauberman, "On the automorphism group of a finite group having no non-identity normal subgroups of odd order." Math.Z. 93, (1966), 154-160.

[5] B. Huppert, N. Blackburn, "Finite Groups III", Gmondlehren der mathematischen 243, Springer - VerZag, Wissenschaften Berlin - Heidelberg - New York, (1982).

[6] M.J. Iranzo, F. Pérez-Monasor, "Existence of $\mathrm{N}$-injectors in a not central normal Fitting class." Israel J. Math. 48, (1984), 123-128. 
[7] M. J. Iranzo, F. Pérez-Monasor, "A class of finite groups having nilpotent injectors." J.Austral.Math.Soc. (to appear).

Department of Mathematics,

Monash University,

clayton,

victoria. 3168,

Australia.

Current Address:

FB. Mathematik der Universität,

Postfach 3980,

D - 6500 Mainz,

F. R. Germany. 